\title{
Ilıman iklim meyve türleri ve sekonder konukçularda bulunan yaprakbiti (Hemiptera: Aphididae) türlerinin parazitoitlenme oranlarının belirlenmesi*
}

\author{
İsmail ALASERHAT ${ }^{\text {ID }}$, Şaban GÜÇLÜ (i)2 \\ 1Bahçe Kültürleri Araştırma Enstitüsü Müdürlüğü, 24060, Erzincan \\ ${ }^{2}$ Ahi Mesut Mah. 1897 Cad. No: 2/16, 06794, Ankara
}

*Bu çalıșma Doktora Tezinden (Atatürk Üniversitesi, Fen Bilimleri Enstitüsü, Bitki Koruma Anabilim Dalı) hazırlanmış olup Tarımsal Araștırmalar ve Politikalar Genel Müdürlüğü (TAGEM- BS-11/04-02/01-18) tarafından desteklenmiștir.

Alınış tarihi: 19 Mart 2020, Kabul tarihi: 25 Kasım 2020

Sorumlu yazar: İsmail ALASERHAT, e-posta: i_alaserhat36@hotmail.com

\section{Öz}

$\mathrm{Bu}$ çalışma, Erzincan ve Gümüşhane illerinde yetiştirilen ılıman iklim meyveleri ve sekonder konukçularda bulunan yaprakbiti türleri ile bunların parazitoitlenme oranlarının belirlenmesi amacıyla 2011-2013 yıllarında yapılmıştır. Örneklemeler, yaprakbiti popülasyonunun yoğun olduğu ilkbahar (mayıs-haziran) ve sonbahar aylarında (eylül-ekim) iki haftada bir, yoğunluğun azaldığı yaz aylarında (temmuz-ağustos) ise ayda bir kez yapılmıștır. Yaprakbiti türlerinin belirlendiği bahçelerden yaprakbiti ile bulaşık ve özellikle de mumyalaşmış yaprakbitlerinin bulunduğu koloniler parazitoit çıkartma kutularına konulmuş, elde edilen toplam parazitli birey sayısı, o örnekte bulunan yaprakbiti sayısına oranlanarak \% parazitoitlenme oranı tespit edilmiștir. Çalıșma sonucunda ılıman iklim meyve türlerinde zarar yapan 12, sekonder konukçularda ise 2 adet olmak üzere toplam 14 yaprakbiti türü ve bunların parazitoitlenme oranları tespit edilmiştir.

Anahtar kelimeler: Yaprakbiti, Parazitoitlenme oranı, Erzincan, Gümüşhane

Determination of parasitism rates of aphid species (Hemiptera: Aphididae) on temperate fruit species and seconder hosts

\section{Abstract}

This study was carried out in 2011-2013, in order to identify the aphid species and their parasitism rates on temperate fruits and seconder hosts grown in
Erzincan and Gümüşhane provinces. The sampling was done every two weeks in spring (May-June) and autumn (September-October) when the aphid population was dense, and once a month in the summer (July-August) when the density decreases. Colonies containing aphids and especially mummified aphids from orchards where aphid species were determined were placed in parasitoid extraction boxes. The number of total parasitic individuals obtained from the colonies including mummy, was compared with the number of total aphids in the sample and the parasitism rate was determined. As a result of the study, a total of 14 aphid species, 12 of which were damaging on temperate fruit species and 2 on secondary hosts, and their parasitism rates were determined.

Key words: Aphid, Parasitism rate, Erzincan, Gümüşhane

\section{Giriş}

Türkiye, coğrafyası itibariyle kültür bitkilerinin yetiştiriciliğine uygunluğunun yanısıra, birçoğunun da gen kaynağı durumundadır. Ülkemiz, meyvecilik açısından önemli bir konuma sahip olup, adeta meyvecilik kültürü ve gen merkezidir (Gerçekçioğlu ve ark., 2008). Biyoçeşitlilik açısından dünyada müstesna bir yeri olan ülkemizde, birçok bitki ve hayvan türünün çok değișik alttür, varyete ve biyotipleri mevcuttur (Ülkümen, 1973).

Türkiye'de 2019 yılı verilerine göre 33.485 .767 da alanda, 20.578.453 ton meyve üretilmiş olup, bunun 168.549 tonu Kuzeydoğu Anadolu Bölgesinde, 
42.138 tonu ise çalışmanın yürütüldüğü Erzincan ilinde üretilmiştir. Doğu Karadeniz Bölgesinde ise 125.029 ton meyve üretimi gerçekleştirilmiş olup bunun 11.796 tonu ise Gümüşhane ilinde üretilmiştir. Erzincan ilindeki bu üretim, içerisinde yer aldığı Kuzeydoğu Anadolu Bölgesi üretim miktarının \%25'ini; Gümüşhane ili ise içerisinde yer aldığı Doğu Karadeniz Bölgesi üretim miktarının \%9.43'ünü içermesi, her iki ilinde meyve yetiştiriciliğine verdiği önemi ortaya koymaktadır (Anonim, 2019).

Birçok üründe olduğu gibi meyve üretiminde de ürün miktarını sınırlayan hastalık, zararlı, yabancı ot vb. birçok etmen bulunmaktadır. Meyvelerde zararlı böcekler Hemiptera, Thysanoptera, Coleoptera, Diptera, Lepidoptera ve Hymenoptera takımları içerisinde yer almaktadır (Özbekve ark., 1998; Gürbüz, 2001; Görür, 2004; Aslan ve Karaca 2005; Narmanlığlu ve Güçlü, 2008; Daşçı ve Güçlü, 2008). Bu zararlılar arasında Hemiptera takımında yer alan yaprakbitlerinin, bitkilerin sürgün, dal, yaprak, meyve, gövde ve köklerinde beslenerek ciddi zarar ve deformasyonlar oluşturdukları, salgıladıkları tatlı maddelerle bitkilerin kirlenmesine, tatlı maddelere yapışan toz ve burada gelişen funguslar nedeniyle de fumajin oluşumuna yol açtıkları; bu oluşumun ise, bitkilerin fotosentez ve solunum kapasitesini azalttığı ifade edilmektedir (Düzgüneș ve Toros, 1978; Lodos, 1986; Ölmez Bayhan ve Ulusoy, 2002). Ayrıca virüs ve benzeri organizmalara da vektörlük ederler ki, çoğu zaman bu şekildeki zararları, diğer zararlarından çok daha önemli olmaktadır. Yaprakbitlerinin bitkisel kökenli 370 virüsün \% 66'sını taşıdıkları belirtilmiştir (Toros, 1973; Matheus, 1993).

Meyvelerde zararlı önemli yaprakbiti türleri Aphis (Linnaeus), Dysaphis (Borner), Hyalopterus (Koch), Myzus (Passerini), Toxoptera (Koch), Pterochloroides (Mordvilko) ve Rhopalosiphum (Koch) cinslerinde yer alırlar (Özbekve ark., 1998; Anonim, 2008). Bu cinsler içerisinden Aphis pomi (De Geer) (Elma yeşil yaprakbiti), Aphis citricola (van der Goot) (Turunçgil yeşil yaprakbiti), Dysaphis devecta (Walker) (Elma kırmızı gal yaprakbiti), Dysaphis plantaginea (Passerini) (Elma gri yaprakbiti), Dysaphis pyri (Boyer de Fonscolombe) (Armut gri yaprakbiti), Hyalopterus pruni (Geoffroy) (Erik unlu yaprakbiti), Myzus cerasi (Fabricius) (Kiraz siyah yaprakbiti), Myzus persicae (Sulzer) (Șeftali yaprakbiti), Toxoptera aurantii (Boyer de Fonscolombe) (Turunçgil siyah yaprakbiti), Pterochloroides persicae (Cholodkovsky) (Şeftali gövde kanlıbiti) ve Rhopalosiphum insertum (Walker) (Elma-ot yaprakbiti) ülkemizde de yoğun olarak görülen önemli meyve zararlısı Aphididae türlerindendir (Özbekve ark., 1998; Gürbüz, 2001; Görür, 2004; Aslan ve Karaca, 2005; Yoldaş ve ark., 2007; Daşçı ve Güçlü, 2008; Narmanlığlu ve Güçlü, 2008; Anonim, 2008; Ölmez Bayhan ve Özdemir, 2009; Alaserhat, 2019; Kaplan, 2019).

Erzincan ve Gümüșhane illerinde yürütülen bu çalışmada, meyve ağaçlarında zarar yapan yaprakbiti türleri ile bunların parazitoitlenme oranları saptanmıştır. Yaprakbiti türleri üzerinde bulunan parazitoitlerin biyolojik mücadelede kullanım imkânları ile ileride yapılacak çalışmalara bir altyapı oluşturmuştur. Böylece, entegre mücadele ve organik meyve yetiştiriciliği kapsamında, yaprakbitleri ile biyolojik mücadelenin temel verileri ortaya konulmuştur.

\section{Materyal ve Yöntem}

\section{Materyal}

Çalışmanın ana materyalini; Erzincan ve Gümüşhane illerinde yetiştirilen ılıman iklim meyve türlerinden elma, armut, ayva, erik, kayısı, şeftali, kiraz, vişne, kızılcık, mahlep, badem ve ceviz ağaçları, sekonder konukçular [(Arctium minus Bernh., Carduus pynocephalus L., Circium arvensis (L.) Scop., Onopordum acanthium L., Onopordum bracteatum Boiss. Et Heldr, Sonchus sp. L. (Asteraceae), Euphorbia virgata Waldst. \& Kit.(, Euphorbiaceae), Malva nicaeensis All. (Malvaceae)] ile bunlar üzerinde bulunan yaprakbiti türleri ve bu türlerin parazitoitleri oluşturmuştur. Ayrıca stereo mikroskop, böcek aspiratörü, parazitoit çıkartma kutuları vb. malzemeler çalışmanın diğer materyallerini oluşturmuştur.

\section{Metot}

\section{Yaprakbiti türlerinin belirlenmesi}

Sürvey çalışmaları 2011-2013 yıllarında, Erzincan Merkeze bağlı Yalnızbağ, Bahçeliköy, Dörtler beldeleri ile Cevizli köyü, Üzümlü Merkez ilçesi ve Bayırbağ beldesi, Kemah Merkez ilçesi, Eriç ve Bozoğlak köyü, İliç ilçesi Merkez, Kemaliye Merkez ilçesi, Yuva ve Toybelen köyü; Gümüşhane Merkez, Pirahmet köyü ve Tekke beldesi ile Torul Merkez ilçesi, Mescitli ve İkisu köylerinde (Şekil 1), Bora ve Karaca (1970)'nın tesadüfi örnekleme yöntemine göre, vejetasyon başlangıcından sezon sonuna kadar 
olan dönemde, üretim alanlarının en az \% 5'inde yürütülmüştür (Çizelge 1). Yaprakbiti türlerinin belirlenmesi amacıyla Erzincan'daki sürvey alanlarından en az ikişer bahçe; Gümüşhane'deki sürvey alanlarından en az birer bahçede Lazarov ve Grigorov (1961) yöntemine göre incelemeler yapılmıştır. Bu metoda göre; 20 ağaca kadar olan bahçelerde ağaçların tamamı, 21-70 ağaç olan bahçelerde 21-30 ağaç, 71-150 ağaç olan bahçelerde 31-40 ağaç, 151-300 ağaç olan bahçelerde 41-80 ağaç, 301-1000 ağaç olan bahçelerde bahçedeki ağaçların \%15, 1000'den fazla ağaç olan bahçelerde ise bahçedeki ağaçların \%5'i kontrol edilmiştir. Örneklemeler, bu bahçelerde bulunan ılıman iklim meyve türlerinden elma, armut, ayva, erik, kayısı, şeftali, kiraz, vişne, kızılcık, mahlep, badem ve ceviz ağaçlarında, mümkün oldukça türlerin birçoğunu içeren bahçelerde yapılmıştır.

Çizelge 1. Erzincan ve Gümüşhane illeri meyve alanları (Anonim 2013) ve incelenen alanlar

\begin{tabular}{cccc}
\hline İl & Lokasyon & $\begin{array}{c}\text { Toplam alan } \\
\text { (da) }\end{array}$ & $\begin{array}{c}\text { İncelenen alan } \\
\text { (da) }\end{array}$ \\
\hline \multirow{3}{*}{ Erzincan } & Merkez & 16.349 & 1.180 \\
& Üzümlü & 10.551 & 910 \\
& Kemah & 3.691 & 262 \\
& İliç & 663 & 45 \\
& Kemaliye & 1.317 & 60 \\
\hline \multirow{2}{*}{ Gümüşhane } & Merkez & 1.478 & 215 \\
& Torul & 1.676 & 220 \\
\hline
\end{tabular}

Yaprakbiti popülasyonunun yoğun olduğu ilkbahar (mayıs-haziran) ve sonbahar aylarında (eylül-ekim) iki haftada bir, yoğunluğun azaldığı yaz aylarında (temmuz-ağustos) ise ayda bir arazi çıkışları yapılmış ağaçların sürgün uçlarından ve yapraklarından örnekler toplanarak kese kâğıtlarına konulup laboratuvara getirilmiş daha sonra bu örnekler içerisinde $\% 70$ etil alkol bulunan ephendorf tüplerine yerleștirilmiştir. Arazi sürveyleri bittikten sonra bu örneklerin preparasyonları yapılmış ve daha sonra yaprakbiti türleri tespit edilmiştir.

\section{Yaprak bitlerinin preparasyonu ve teşhisi}

Yaprakbiti preparasyonlarının yapımında Hille Ris Lambers (1950)'in yöntemi kullanılmıştır. Yaprakbitlerinin teşhisinde; Remaudiere (1954), Bodenheimer ve Swirski (1957), Bissel (1978), Blackman ve Eastop (1984, 1994, 2000, 2020), Eastop (1971, 1972), Hille Ris Lambers (1945, 1947a, 1947b, 1949, 1969, 1973), Tuatay ve Remaudiere (1964), Shaposhnikov (1964), Stroyan $(1961,1963,1977,1984)$ 'dan ve Nazife Tuatay Bitki
Koruma Müzesindeki teşhisli örneklerden yararlanılmıștır.

Yaprakbitlerinin sistematik olarak sinfflandırması, geçerli olan son isim ve sinonimlerinin tespitinde Eastop ve Hille Ris Lambers (1976), Remaudiere ve Remaudiere (1997) esas alınmıștır.

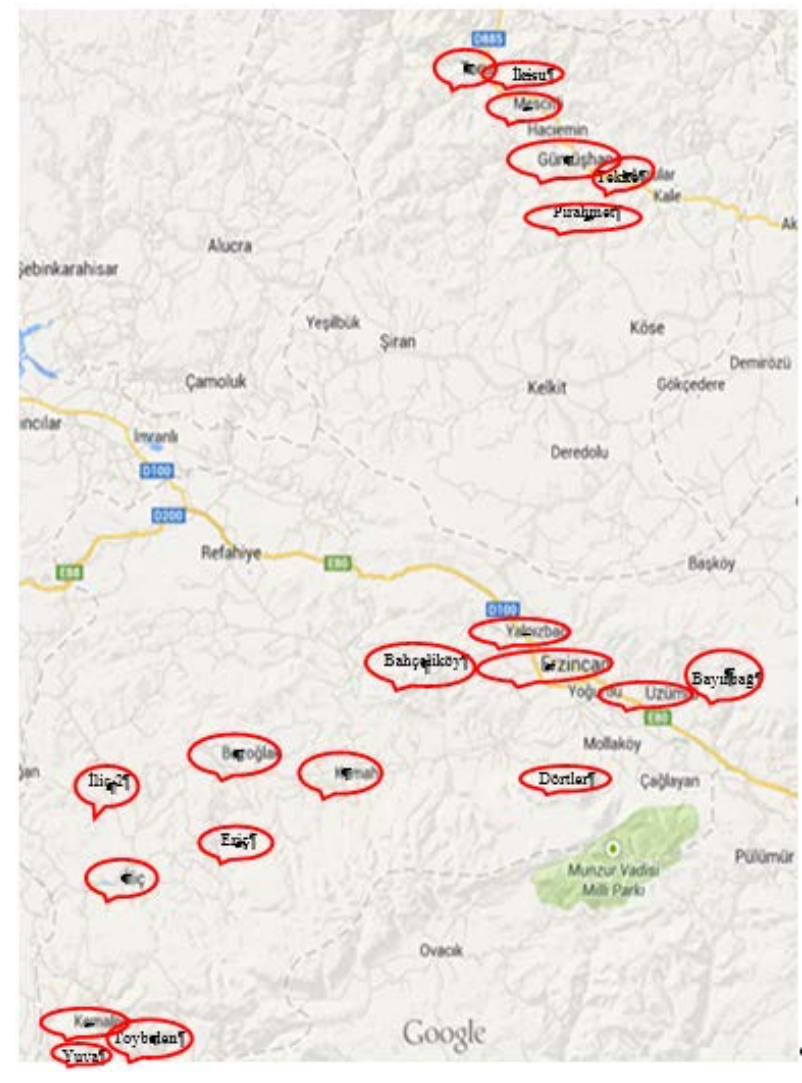

Şekil 1. Erzincan ve Gümüşhane illerinde çalışmaların yürütüldüğü alanlar

\section{Parazitoitlenme oranlarının belirlenmesi}

Yaprakbiti parazitoitlerini belirlenmek maksadiyla, sürveylerin yapıldı̆̆ bahçelerden içerisinde mumyalașmıș yaprakbitlerinin bulunduğu koloniler kese kâğıtlarına konulmuș ve buz kutularına yerleştirilerek laboratuvara getirilmiștir. Laboratuvara getirilen bu örneklerde öncelikle yaprakbiti dışındaki tüm zararlılar temizlenmiş, daha sonra ucu dışarıda olacak şekilde bir cam tüp yerleştirilen parazitoit çlkartma kutularına konulmuştur. Parazitoit çıkartma kutuları günlük olarak kontrol edilmiş, cam tüpte toplanan parazitoit bireyler alınmış, teşhis edilmek üzere içerisinde \%95'lik etil alkol bulunan ephendorf tüplere aktarılmıştır (Yumruktepe, 1993).

Elde edilen toplam parazitli birey sayısı, o örnekte bulunan yaprakbiti sayısına oranlanarak \% 
parazitoitlenme oranı tespit edilmiștir (Praslicka ve Huszar, 2005).

\section{Bulgular}

Çalışmalar esnasinda yaprakbitlerinin parazitoitlerinin belirlenmesi amaciyla mumya örnekler alındıkları tarih ve toplam yaprakbiti sayıları da kaydedilerek laboratuvarda kültüre alınmış, arazi çalışmaları bittikten sonra parazitoit çıkartma kutuları açlarak, elde edilen toplam parazitli birey (mumya) sayısı, o örnekte bulunan yaprakbiti sayısına oranlanarak \% parazitoitlenme oranı tespit edilmiștir. 2011 yılı parazitoitlenme oranları Çizelge 2'de verilmiștir.

Çizelge 2. 2011 yılında elde edilen parazitoitlenmiş yaprakbiti sayıları, konukçuları ve parazitoitlenme oranlarının saptanması

\begin{tabular}{|c|c|c|c|c|c|}
\hline Tarih & Yaprakbiti türü & $\begin{array}{l}\text { Toplam } \\
\text { yaprakbiti } \\
\text { sayısı }\end{array}$ & $\begin{array}{c}\text { Toplam } \\
\text { parazitoitlenmiş } \\
\text { (mumya) yaprakbiti } \\
\text { sayısı } \\
\end{array}$ & $\begin{array}{c}\text { Parazitoitlenme } \\
\text { oranları } \\
(\%)\end{array}$ & $\begin{array}{c}\text { Elde edilen } \\
\text { parazitoit } \\
\text { sayısı }\end{array}$ \\
\hline 04.05 .2011 & $\begin{array}{l}\text { Dysaphis (Pomaphis) pyri (Boyer de } \\
\text { Fonscolombe) }\end{array}$ & 44 & 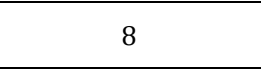 & 18.2 & 6 \\
\hline 06.05 .2011 & $\begin{array}{c}\text { Dysaphis (Pomaphis) pyri (Boyer de } \\
\text { Fonscolombe) }\end{array}$ & 52 & 2 & 3.8 & 1 \\
\hline 09.05 .2011 & $\begin{array}{c}\text { Dysaphis (Pomaphis) pyri (Boyer de } \\
\text { Fonscolombe) }\end{array}$ & 45 & 10 & 22.2 & 4 \\
\hline 09.05.2011 & $\begin{array}{c}\text { Dysaphis (Pomaphis) pyri (Boyer de } \\
\text { Fonscolombe) }\end{array}$ & 21 & 2 & 9.5 & 0 \\
\hline 09.05.2011 & Dysaphis devecta (Walker) & 12 & 0 & 0 & 0 \\
\hline 09.05.2011 & $\begin{array}{c}\text { Dysaphis (Pomaphis) pyri (Boyer de } \\
\text { Fonscolombe) }\end{array}$ & 32 & 1 & 3.1 & 0 \\
\hline 09.05 .2011 & Myzus cerasi (Fabricius) & 14 & 0 & 0 & 0 \\
\hline 10.05 .2011 & Dysaphis devecta (Walker) & 37 & 1 & 2.7 & 0 \\
\hline 10.05.2011 & Dysaphis devecta (Walker) & 19 & 0 & 0 & 0 \\
\hline 10.05 .2011 & $\begin{array}{c}\text { Dysaphis (Pomaphis) pyri (Boyer de } \\
\text { Fonscolombe) }\end{array}$ & 5 & 1 & 20 & 0 \\
\hline 10.05 .2011 & $\begin{array}{c}\text { Dysaphis (Pomaphis) pyri (Boyer de } \\
\text { Fonscolombe) }\end{array}$ & 72 & 0 & 0 & 0 \\
\hline 20.05 .2011 & Dysaphis devecta (Walker) & 33 & 3 & 9.1 & 0 \\
\hline 20.05 .2011 & Dysaphis devecta (Walker) & 26 & 0 & 0 & 0 \\
\hline 23.05 .2011 & Brachycaudus (Prunaphis) cardui (L.) & 11 & 3 & 27.3 & 1 \\
\hline 23.05 .2011 & Dysaphis devecta (Walker) & 26 & 0 & 0 & 0 \\
\hline 23.05 .2011 & Dysaphis devecta (Walker) & 10 & 1 & 10 & 1 \\
\hline 23.05.2011 & Dysaphis devecta (Walker) & 2 & 1 & 50 & 0 \\
\hline 23.05.2011 & Dysaphis devecta (Walker) & 41 & 2 & 4.9 & 0 \\
\hline 23.05 .2011 & Dysaphis devecta (Walker) & 16 & 3 & 18.8 & 1 \\
\hline 23.05.2011 & $\begin{array}{c}\text { Dysaphis (Pomaphis) pyri (Boyer de } \\
\text { Fonscolombe) }\end{array}$ & 20 & 0 & 0 & 0 \\
\hline 23.05.2011 & Dysaphis devecta (Walker) & 37 & 0 & 0 & 0 \\
\hline 23.05.2011 & Dysaphis devecta (Walker) & 11 & 0 & 0 & 0 \\
\hline 26.05 .2011 & Dysaphis devecta (Walker) & 18 & 0 & 0 & 0 \\
\hline 26.05 .2011 & Myzus cerasi (Fabricius) & 54 & 0 & 0 & 0 \\
\hline 26.05.2011 & $\begin{array}{c}\text { Dysaphis (Pomaphis) pyri (Boyer de } \\
\text { Fonscolombe) }\end{array}$ & 120 & 0 & 0 & 0 \\
\hline 26.05.2011 & $\begin{array}{c}\text { Dysaphis (Pomaphis) pyri (Boyer de } \\
\text { Fonscolombe) } \\
\end{array}$ & 48 & 0 & 0 & 0 \\
\hline 26.05 .2011 & Dysaphis devecta (Walker) & 81 & 0 & 0 & 0 \\
\hline 26.05.2011 & $\begin{array}{c}\text { Myzus (Nectarosiphon) persicae } \\
\text { (Sulzer) }\end{array}$ & 26 & 1 & 3.8 & 0 \\
\hline 27.05.2011 & $\begin{array}{c}\text { Dysaphis (Pomaphis) plantaginea } \\
\text { (Passerini) }\end{array}$ & 28 & 16 & 57.1 & 7 \\
\hline 27.05 .2011 & $\begin{array}{c}\text { Dysaphis (Pomaphis) pyri (Boyer de } \\
\text { Fonscolombe) }\end{array}$ & 34 & 0 & 0 & 0 \\
\hline 27.05.2011 & $\begin{array}{c}\text { Dysaphis (Pomaphis) pyri (Boyer de } \\
\text { Fonscolombe) }\end{array}$ & 220 & 15 & 6.8 & 7 \\
\hline 27.05.2011 & Hyalopterus pruni (Geoffroy) & 300 & 0 & 0 & 0 \\
\hline
\end{tabular}


Çizelge 2'nin devamı

\begin{tabular}{|c|c|c|c|c|c|}
\hline 27.05 .2011 & Myzus cerasi (Fabricius) & 93 & 0 & 0 & 0 \\
\hline 27.05 .2011 & Dysaphis devecta & 10 & 5 & 50 & 0 \\
\hline 01.06.2011 & $\begin{array}{c}\text { Dysaphis (Pomaphis) pyri (Boyer de } \\
\text { Fonscolombe) }\end{array}$ & 7 & 0 & 0 & 0 \\
\hline 06.06 .2011 & Dysaphis devecta (Walker) & 83 & 9 & 10.8 & 5 \\
\hline 06.06 .2011 & Myzus cerasi (Fabricius) & 52 & 0 & 0 & 0 \\
\hline 06.06 .2011 & Brachycaudus helichrysi (Kaltenbach) & 41 & 0 & 0 & 0 \\
\hline 06.06 .2011 & Dysaphis devecta (Walker) & 21 & 10 & 47.6 & 5 \\
\hline 06.06 .2011 & $\begin{array}{c}\text { Dysaphis (Pomaphis) pyri (Boyer de } \\
\text { Fonscolombe) }\end{array}$ & 104 & 8 & 7.7 & 1 \\
\hline 06.06 .2011 & Dysaphis devecta (Walker) & 94 & 15 & 16 & 9 \\
\hline 06.06 .2011 & Brachycaudus (Prunaphis) cardui (L.) & 22 & 0 & 0 & 0 \\
\hline 06.06 .2011 & Aphis pomi De Geer & 28 & 0 & 0 & 0 \\
\hline 06.06 .2011 & $\begin{array}{l}\text { Brachycaudus (Thuleaphis) } \\
\text { amygdalinus (Schouteden) }\end{array}$ & 63 & 0 & 0 & 0 \\
\hline 06.06 .2011 & Dysaphis devecta (Walker) & 63 & 6 & 9.5 & 6 \\
\hline 08.06.2011 & $\begin{array}{c}\text { Myzus (Nectarosiphon) persicae } \\
\text { (Sulzer) }\end{array}$ & 88 & 0 & 0 & 0 \\
\hline 08.06 .2011 & Hyalopterus pruni (Geoffroy) & 14 & 0 & 0 & 0 \\
\hline 08.06 .2011 & Dysaphis devecta (Walker) & 17 & 2 & 11.8 & 0 \\
\hline 08.06 .2011 & Myzus cerasi (Fabricius) & 580 & 0 & 0 & 0 \\
\hline 08.06.2011 & Aphis pomi De Geer & 10 & 2 & 20 & 0 \\
\hline 08.06.2011 & Dysaphis devecta (Walker) & 64 & 0 & 0 & 0 \\
\hline 08.06 .2011 & $\begin{array}{c}\text { Dysaphis (Pomaphis) pyri (Boyer de } \\
\text { Fonscolombe) }\end{array}$ & 71 & 9 & 12.7 & 4 \\
\hline 08.06.2011 & Hyalopterus pruni (Geoffroy) & 115 & 0 & 0 & 0 \\
\hline 09.06.2011 & Hyalopterus pruni (Geoffroy) & 4 & 1 & 25 & 0 \\
\hline 09.06 .2011 & Myzus cerasi (Fabricius) & 310 & 0 & 0 & 0 \\
\hline 09.06 .2011 & Brachycaudus helichrysi (Kaltenbach) & 340 & 1 & 0.3 & 0 \\
\hline 20.06 .2011 & Hyalopterus pruni (Geoffroy) & 124 & 10 & 8.1 & 0 \\
\hline 20.06 .2011 & Brachycaudus helichrysi (Kaltenbach) & 210 & 2 & 0.9 & 0 \\
\hline 20.06 .2011 & Hyalopterus pruni (Geoffroy) & 29 & 0 & 0 & 0 \\
\hline 22.06.2011 & $\begin{array}{c}\text { Dysaphis (Pomaphis) plantaginea } \\
\text { (Passerini) }\end{array}$ & 51 & 4 & 7.8 & 3 \\
\hline 22.06 .2011 & Myzus cerasi (Fabricius) & 1200 & 0 & 0 & 0 \\
\hline 22.06 .2011 & Hyalopterus pruni (Geoffroy) & 50 & 2 & 4 & 0 \\
\hline 22.06 .2011 & Hyalopterus pruni (Geoffroy) & 34 & 1 & 2.9 & 0 \\
\hline 22.06 .2011 & Hyalopterus pruni (Geoffroy) & 280 & 0 & 0 & 0 \\
\hline 24.06 .2011 & Aphis pomi De Geer & 175 & 0 & 0 & 0 \\
\hline 24.06 .2011 & Hyalopterus pruni (Geoffroy) & 103 & 8 & 7.8 & 0 \\
\hline 24.06 .2011 & Hyalopterus pruni (Geoffroy) & 81 & 24 & 29.6 & 2 \\
\hline 24.06 .2011 & $\begin{array}{l}\text { Dysaphis (Pomaphis) pyri (Boyer de } \\
\text { Fonscolombe) }\end{array}$ & 35 & 0 & 0 & 0 \\
\hline 24.06 .2011 & Dysaphis devecta (Walker) & 68 & 5 & 7.4 & 1 \\
\hline 24.06 .2011 & Myzus cerasi (Fabricius) & 240 & 11 & 4.6 & 5 \\
\hline 27.06 .2011 & Dysaphis devecta (Walker) & 82 & 9 & 1.1 & 6 \\
\hline 27.06 .2011 & Myzus cerasi (Fabricius) & 295 & 6 & 2.1 & 0 \\
\hline 27.06 .2011 & $\begin{array}{c}\text { Myzus (Nectarosiphon) persicae } \\
\text { (Sulzer) }\end{array}$ & 28 & 0 & 0 & 0 \\
\hline 27.06 .2011 & Hyalopterus pruni (Geoffroy) & 210 & 3 & 1.4 & 2 \\
\hline 27.06 .2011 & Brachycaudus helichrysi (Kaltenbach) & 36 & 4 & 11.1 & 0 \\
\hline 27.06 .2011 & Dysaphis devecta (Walker) & 36 & 1 & 2.8 & 0 \\
\hline 25.07 .2011 & Myzus cerasi (Fabricius) & 125 & 0 & 0 & 0 \\
\hline 25.07 .2011 & $\begin{array}{c}\text { Dysaphis (Pomaphis) pyri (Boyer de } \\
\text { Fonscolombe) }\end{array}$ & 19 & 1 & 5.3 & 0 \\
\hline 25.07 .2011 & Hyalopterus pruni (Geoffroy) & 92 & 7 & 7.6 & 0 \\
\hline 27.07 .2011 & Hyalopterus pruni (Geoffroy) & 74 & 16 & 21.6 & 1 \\
\hline
\end{tabular}


Çizelge 2'nin devamı

\begin{tabular}{|c|c|c|c|c|c|}
\hline 16.08.2011 & Aphis pomi De Geer & 650 & 0 & 0 & 0 \\
\hline 16.08 .2011 & Aphis pomi De Geer & 148 & 0 & 0 & 0 \\
\hline 17.08 .2011 & Aphis pomi De Geer & 154 & 5 & 3.2 & 3 \\
\hline 17.08.2011 & Myzus cerasi (Fabricius) & 345 & 0 & 0 & 0 \\
\hline 17.08.2011 & Hyalopterus pruni (Geoffroy) & 375 & 0 & 0 & 0 \\
\hline 17.08 .2011 & Hyalopterus pruni (Geoffroy) & 195 & 6 & 3.1 & 0 \\
\hline 19.08 .2011 & Aphis pomi De Geer & 210 & 0 & 0 & 0 \\
\hline 19.08.2011 & Hyalopterus pruni (Geoffroy) & 94 & 7 & 7.4 & 0 \\
\hline 19.08.2011 & Myzus cerasi (Fabricius) & 59 & 0 & 0 & 0 \\
\hline 19.08.2011 & Aphis pomi De Geer & 280 & 4 & 1.4 & 2 \\
\hline 21.09 .2011 & Myzus cerasi (Fabricius) & 380 & 7 & 1.8 & 3 \\
\hline 21.09 .2011 & Aphis pomi De Geer & 410 & 0 & 0 & 0 \\
\hline 23.09 .2011 & Hyalopterus pruni (Geoffroy) & 210 & 0 & 0 & 0 \\
\hline 23.09.2011 & $\begin{array}{l}\text { Brachycaudus (Thuleaphis) } \\
\text { amygdalinus (Schouteden) }\end{array}$ & 81 & 0 & 0 & 0 \\
\hline 05.10 .2011 & Hyalopterus pruni (Geoffroy) & 140 & 3 & 2.1 & 2 \\
\hline 06.10 .2011 & $\begin{array}{c}\text { Pterochloroides persicae } \\
\text { (Cholodkovsky) }\end{array}$ & 186 & 0 & 0 & 0 \\
\hline 18.10.2011 & $\begin{array}{c}\text { Pterochloroides persicae } \\
\text { (Cholodkovsky) }\end{array}$ & 175 & 0 & 0 & 0 \\
\hline 19.10 .2011 & Myzus cerasi (Fabricius) & 365 & 0 & 0 & 0 \\
\hline 21.10 .2011 & Hyalopterus pruni (Geoffroy) & 38 & 0 & 0 & 0 \\
\hline
\end{tabular}

Çizelge incelendiğinde 2011 yılında genel parazitoitlenme oranı düşük, \%6.3 olarak tespit edilmiştir. Örnek alınan tüm meyve türleri içerisinde yüzde parazitoitlenmenin en yüksek olduğu türler ise sirasıyla Dysaphis (Pomaphis) plantaginea (\%32.45), Brachycaudus (Acaudus) cardui (\%13.65), Dysaphis devecta (\%10.30), Dysaphis (Pomaphis) pyri
(\%6.43) ve Hyalopterus pruni (\%4.62) olmuştur. Ayrica en fazla parazitoitlenme 27.05.2011 tarihinde elma üzerinden elde edilen Dysaphis (Pomaphis) plantaginea'da olmuştur. Bu örnekte parazitoitlenme oranı \%57.1 olmuştur. Çalışmamızın 2012 yılı parazitoitlenme oranları Çizelge 3'de verilmiştir.

Çizelge 3. 2012 yılında elde edilen parazitoitlenmiş yaprakbiti sayıları, konukçuları ve parazitoitlenme oranlarının saptanması

\begin{tabular}{|c|c|c|c|c|c|}
\hline Tarih & Yaprakbiti türü & $\begin{array}{c}\text { Toplam } \\
\text { yaprakbiti sayısı }\end{array}$ & $\begin{array}{c}\text { Toplam } \\
\text { parazitoitlenmiş } \\
\text { (mumya) } \\
\text { yaprakbiti sayısı }\end{array}$ & $\begin{array}{l}\text { Parazitoitlenme oranları } \\
\qquad \%)\end{array}$ & $\begin{array}{c}\text { Elde edilen } \\
\text { parazitoit } \\
\text { sayısı }\end{array}$ \\
\hline 07.05 .2012 & Brachycaudus helichrysi (Kaltenbach) & 65 & 7 & 10.77 & 4 \\
\hline 09.05 .2012 & $\begin{array}{c}\text { Dysaphis (Pomaphis) pyri (Boyer de } \\
\text { Fonscolombe) }\end{array}$ & 310 & 17 & 5.48 & 0 \\
\hline 09.05.2012 & $\begin{array}{c}\text { Dysaphis (Pomaphis) pyri (Boyer de } \\
\text { Fonscolombe) }\end{array}$ & 79 & 13 & 16.46 & 1 \\
\hline 09.05.2012 & Brachycaudus helichrysi (Kaltenbach) & 290 & 32 & 11.03 & 0 \\
\hline 11.05.2012 & $\begin{array}{c}\text { Dysaphis (Pomaphis) pyri (Boyer de } \\
\text { Fonscolombe) }\end{array}$ & 149 & 14 & 9.40 & 8 \\
\hline 11.05 .2012 & Dysaphis devecta (Walker) & 42 & 2 & 4.76 & 0 \\
\hline 11.05 .2012 & $\begin{array}{c}\text { Myzus (Nectarosiphon) persicae } \\
\text { (Sulzer) }\end{array}$ & 350 & 6 & 1.71 & 0 \\
\hline 14.05.2012 & $\begin{array}{c}\text { Dysaphis (Pomaphis) pyri (Boyer de } \\
\text { Fonscolombe) }\end{array}$ & 27 & 7 & 29.17 & 7 \\
\hline 14.05 .2012 & Dysaphis devecta (Walker) & 147 & 21 & 14.29 & 12 \\
\hline 14.05 .2012 & Brachycaudus helichrysi (Kaltenbach) & 68 & 2 & 2.94 & 0 \\
\hline 16.05 .2012 & $\begin{array}{c}\text { Dysaphis (Pomaphis) pyri (Boyer de } \\
\text { Fonscolombe) }\end{array}$ & 400 & 26 & 6.50 & 3 \\
\hline 17.05 .2012 & $\begin{array}{c}\text { Dysaphis (Pomaphis) pyri (Boyer de } \\
\text { Fonscolombe) }\end{array}$ & 340 & 21 & 6.18 & 4 \\
\hline 17.05 .2012 & $\begin{array}{c}\text { Dysaphis (Pomaphis) pyri (Boyer de } \\
\text { Fonscolombe) }\end{array}$ & 102 & 4 & 3.92 & 2 \\
\hline
\end{tabular}


Çizelge 3'ün devamı

\begin{tabular}{|c|c|c|c|c|c|}
\hline 17.05.2012 & $\begin{array}{l}\text { Dysaphis (Pomaphis) pyri (Boyer de } \\
\text { Fonscolombe) }\end{array}$ & 152 & 9 & 5.92 & 1 \\
\hline 17.05 .2012 & Hyalopterus pruni (Geoffroy) & 90 & 10 & 11.11 & 5 \\
\hline 17.05 .2012 & Brachycaudus helichrysi (Kaltenbach) & 160 & 6 & 3.75 & 0 \\
\hline 21.05 .2012 & Dysaphis devecta (Walker) & 60 & 5 & 8.33 & 1 \\
\hline 21.05 .2012 & Brachycaudus helichrysi (Kaltenbach) & 22 & 2 & 9.09 & 2 \\
\hline 21.05 .2012 & Brachycaudus helichrysi (Kaltenbach) & 280 & 14 & 5.00 & 0 \\
\hline 21.05 .2012 & Myzus cerasi (Fabricius) & 108 & 5 & 4.63 & 1 \\
\hline 21.05 .2012 & Myzus cerasi (Fabricius) & 150 & 4 & 2.67 & 1 \\
\hline 23.05 .2012 & $\begin{array}{c}\text { Dysaphis (Pomaphis) pyri (Boyer de } \\
\text { Fonscolombe) }\end{array}$ & 410 & 16 & 3.90 & 0 \\
\hline 23.05 .2012 & Dysaphis devecta (Walker) & 308 & 22 & 7.14 & 0 \\
\hline 24.05 .2012 & Brachycaudus helichrysi (Kaltenbach) & 625 & 25 & 4.00 & 25 \\
\hline 24.05 .2012 & Hyalopterus pruni (Geoffroy) & 1100 & 69 & 6.27 & 54 \\
\hline 25.05 .2012 & $\begin{array}{c}\text { Dysaphis (Pomaphis) pyri (Boyer de } \\
\text { Fonscolombe) }\end{array}$ & 340 & 17 & 5 & 4 \\
\hline 25.05 .2012 & Dysaphis devecta (Walker) & 272 & 20 & 7.35 & 0 \\
\hline 25.05 .2012 & Brachycaudus helichrysi (Kaltenbach) & 110 & 9 & 8.18 & 1 \\
\hline 28.05 .2012 & $\begin{array}{c}\text { Dysaphis (Pomaphis) pyri (Boyer de } \\
\text { Fonscolombe) }\end{array}$ & 14 & 9 & 64.29 & 7 \\
\hline 28.05 .2012 & $\begin{array}{c}\text { Dysaphis (Pomaphis) pyri (Boyer de } \\
\text { Fonscolombe) }\end{array}$ & 84 & 5 & 5.95 & 2 \\
\hline 28.05.2012 & $\begin{array}{c}\text { Dysaphis (Pomaphis) pyri (Boyer de } \\
\text { Fonscolombe) }\end{array}$ & 44 & 7 & 15.91 & 6 \\
\hline 28.05 .2012 & Dysaphis devecta (Walker) & 120 & 11 & 9.17 & 3 \\
\hline 28.05 .2012 & Brachycaudus helichrysi (Kaltenbach) & 99 & 14 & 14.14 & 0 \\
\hline 28.05 .2012 & Brachycaudus helichrysi (Kaltenbach) & 185 & 2 & 1.08 & 0 \\
\hline 30.05 .2012 & $\begin{array}{c}\text { Dysaphis (Pomaphis) pyri (Boyer de } \\
\text { Fonscolombe) }\end{array}$ & 284 & 18 & 6.34 & 3 \\
\hline 30.05 .2012 & $\begin{array}{l}\text { Dysaphis (Pomaphis) pyri (Boyer de } \\
\text { Fonscolombe) }\end{array}$ & 29 & 2 & 6.90 & 0 \\
\hline 30.05 .2012 & $\begin{array}{l}\text { Dysaphis (Pomaphis) pyri (Boyer de } \\
\text { Fonscolombe) }\end{array}$ & 108 & 11 & 10.19 & 9 \\
\hline 30.05 .2012 & $\begin{array}{c}\text { Dysaphis (Pomaphis) pyri (Boyer de } \\
\text { Fonscolombe) }\end{array}$ & 310 & 10 & 3.23 & 3 \\
\hline 30.05 .2012 & Dysaphis devecta (Walker) & 65 & 12 & 18.46 & 0 \\
\hline 30.05 .2012 & Myzus cerasi (Fabricius) & 54 & 2 & 3.70 & 0 \\
\hline 01.06 .2012 & Dysaphis devecta (Walker) & 86 & 7 & 8.14 & 0 \\
\hline 01.06 .2012 & Hyalopterus pruni (Geoffroy) & 368 & 17 & 4.62 & 17 \\
\hline 11.06 .2012 & $\begin{array}{c}\text { Dysaphis (Pomaphis) pyri (Boyer de } \\
\text { Fonscolombe) }\end{array}$ & 68 & 15 & 22.06 & 12 \\
\hline 11.06 .2012 & Brachycaudus helichrysi (Kaltenbach) & 221 & 4 & 1.81 & 0 \\
\hline 11.06 .2012 & Microlophium carnosum (Buckton) & 70 & 45 & 64.29 & 23 \\
\hline 13.06 .2012 & $\begin{array}{c}\text { Dysaphis (Pomaphis) pyri (Boyer de } \\
\text { Fonscolombe) }\end{array}$ & 36 & 3 & 8.33 & 2 \\
\hline 13.06 .2012 & Eriosoma lanigerum (Hausmann) & 60 & 45 & 75.00 & 30 \\
\hline 13.06 .2012 & Eriosoma lanigerum (Hausmann) & 345 & 318 & 92.17 & 120 \\
\hline 13.06 .2012 & Hyalopterus pruni (Geoffroy) & 390 & 10 & 2.56 & 9 \\
\hline 15.06 .2012 & Hyalopterus pruni (Geoffroy) & 286 & 9 & 3.15 & 2 \\
\hline 18.06 .2012 & Dysaphis devecta (Walker) & 76 & 9 & 11.84 & 6 \\
\hline 18.06 .2012 & Hyalopterus pruni (Geoffroy) & 382 & 74 & 19.37 & 35 \\
\hline 18.06 .2012 & Hyalopterus pruni (Geoffroy) & 475 & 9 & 1.89 & 0 \\
\hline 18.06 .2012 & Hyalopterus pruni (Geoffroy) & 116 & 4 & 3.45 & 3 \\
\hline 18.06 .2012 & Hyalopterus pruni (Geoffroy) & 198 & 7 & 3.54 & 0 \\
\hline 25.06 .2012 & Aphis pomi De Geer & 106 & 13 & 12.26 & 8 \\
\hline 04.07 .2012 & Hyalopterus pruni (Geoffroy) & 148 & 5 & 3.38 & 1 \\
\hline 18.07 .2012 & $\begin{array}{c}\text { Dysaphis (Pomaphis) pyri (Boyer de } \\
\text { Fonscolombe) }\end{array}$ & 83 & 5 & 6.02 & 2 \\
\hline
\end{tabular}


Çizelge 3'ün devamı

\begin{tabular}{|c|c|c|c|c|c|}
\hline 18.07.2012 & Dysaphis (Pomaphis) pyri (Boyer de Fonscolombe) & 9 & 1 & 11.11 & 0 \\
\hline 18.07.2012 & Aphis pomi De Geer & 86 & 1 & 1.16 & 1 \\
\hline 18.07.2012 & Dysaphis devecta (Walker) & 22 & 6 & 27.27 & 2 \\
\hline 18.07.2012 & Aphis pomi De Geer & 320 & 4 & 1.25 & 4 \\
\hline 18.07.2012 & Eriosoma lanigerum (Hausmann) & 104 & 91 & 87.50 & 70 \\
\hline 18.07.2012 & Eriosoma lanigerum (Hausmann) & 80 & 72 & 90.00 & 23 \\
\hline 18.07.2012 & Eriosoma lanigerum (Hausmann) & 103 & 42 & 40.78 & 11 \\
\hline 18.07.2012 & Hyalopterus pruni (Geoffroy) & 126 & 3 & 2.38 & 2 \\
\hline 18.07.2012 & Brachycaudus helichrysi (Kaltenbach) & 128 & 33 & 25.78 & 19 \\
\hline 18.07.2012 & Hyalopterus pruni (Geoffroy) & 154 & 2 & 1.30 & 0 \\
\hline 18.07.2012 & Hyalopterus pruni (Geoffroy) & 162 & 12 & 7.41 & 9 \\
\hline 18.07.2012 & Hyalopterus pruni (Geoffroy) & 122 & 1 & 0.82 & 1 \\
\hline 18.07.2012 & Hyalopterus pruni (Geoffroy) & 246 & 2 & 0.81 & 2 \\
\hline 18.07.2012 & Hyalopterus pruni (Geoffroy) & 530 & 11 & 2.08 & 5 \\
\hline 18.07.2012 & Hyalopterus pruni (Geoffroy) & 490 & 36 & 7.35 & 9 \\
\hline 20.07.2012 & Dysaphis devecta (Walker) & 66 & 2 & 1.52 & 2 \\
\hline 20.07.2012 & Hyalopterus pruni (Geoffroy) & 460 & 23 & 5.00 & 8 \\
\hline 20.07.2012 & Hyalopterus pruni (Geoffroy) & 26 & 1 & 3.85 & 1 \\
\hline 20.07.2012 & Hyalopterus pruni (Geoffroy) & 45 & 4 & 8.89 & 2 \\
\hline 20.07.2012 & Hyalopterus pruni (Geoffroy) & 56 & 1 & 1.79 & 0 \\
\hline 20.07.2012 & Hyalopterus pruni (Geoffroy) & 560 & 3 & 0.54 & 3 \\
\hline 20.07.2012 & Hyalopterus pruni (Geoffroy) & 165 & 4 & 2.42 & 2 \\
\hline 20.07.2012 & Hyalopterus pruni (Geoffroy) & 214 & 2 & 0.93 & 0 \\
\hline 23.07.2012 & Hyalopterus pruni (Geoffroy) & 28 & 1 & 3.57 & 0 \\
\hline 06.08 .2012 & Hyalopterus pruni (Geoffroy) & 45 & 1 & 2.22 & 1 \\
\hline 08.08.2012 & Dysaphis (Pomaphis) plantaginea (Passerini) & 120 & 102 & 85.00 & 66 \\
\hline 08.08 .2012 & Aphis craccivora Koch & 114 & 32 & 28.07 & 32 \\
\hline 08.08.2012 & Hyalopterus pruni (Geoffroy) & 80 & 2 & 2.50 & 0 \\
\hline 04.09 .2012 & Aphis pomi De Geer & 210 & 5 & 2.38 & 5 \\
\hline 05.09 .2012 & Hyalopterus pruni (Geoffroy) & 28 & 2 & 7.14 & 1 \\
\hline 05.09 .2012 & Hyalopterus pruni (Geoffroy) & 86 & 2 & 2.33 & 0 \\
\hline 07.09 .2012 & Aphis pomi De Geer & 51 & 3 & 5.88 & 1 \\
\hline 07.09.2012 & Hyalopterus pruni (Geoffroy) & 65 & 2 & 3.08 & 2 \\
\hline 09.09.2012 & Eriosoma lanigerum (Hausmann) & 340 & 315 & 92.68 & 104 \\
\hline 18.09.2012 & Aphis pomi De Geer & 580 & 6 & 1.03 & 4 \\
\hline 21.09 .2012 & Hyalopterus pruni (Geoffroy) & 10 & 1 & 10.00 & 0 \\
\hline 21.09 .2012 & Uroleucon sonchi (L.) & 112 & 2 & 1.79 & 2 \\
\hline 17.10 .2012 & Eriosoma lanigerum (Hausmann) & 90 & 78 & 86.67 & 0 \\
\hline 17.10.2012 & Hyalopterus pruni (Geoffroy) & 5 & 1 & 20.00 & 0 \\
\hline
\end{tabular}

Çizelge 4. 2013 yılında elde edilen parazitoitlenmiş yaprakbiti sayıları, konukçuları ve parazitoitlenme oranlarının saptanması

\begin{tabular}{|c|c|c|c|c|c|}
\hline Tarih & Yaprakbiti türü & $\begin{array}{c}\text { Toplam } \\
\text { yaprakbiti } \\
\text { sayısı }\end{array}$ & $\begin{array}{c}\text { Toplam } \\
\text { parazitoitlenmiş } \\
\text { (mumya) yaprakbiti } \\
\text { sayısı }\end{array}$ & $\begin{array}{c}\text { Parazitoitlenme } \\
\text { oranları } \\
(\%)\end{array}$ & $\begin{array}{c}\text { Elde edilen } \\
\text { parazitoit } \\
\text { sayısı }\end{array}$ \\
\hline 07.05 .2013 & Dysaphis devecta (Walker) & 172 & 9 & 5.23 & 5 \\
\hline 07.05 .2013 & Dysaphis devecta (Walker) & 89 & 7 & 7.87 & 3 \\
\hline 07.05 .2013 & Hyalopterus pruni (Geoffroy) & 68 & 7 & 10.29 & 4 \\
\hline 08.05 .2013 & $\begin{array}{c}\text { Dysaphis (Pomaphis) pyri (Boyer de } \\
\text { Fonscolombe) }\end{array}$ & 85 & 6 & 7.06 & 5 \\
\hline 13.05 .2013 & $\begin{array}{c}\text { Chromaphis juglandicola } \\
\text { (Kaltenbach) }\end{array}$ & 105 & 2 & 1.90 & 1 \\
\hline 13.05 .2013 & Hyalopterus pruni (Geoffroy) & 248 & 5 & 2.02 & 5 \\
\hline 21.05 .2013 & Brachycaudus (Prunaphis) cardui (L.) & 55 & 2 & 3.64 & 2 \\
\hline
\end{tabular}


Çizelge 4'ün devamı

\begin{tabular}{|c|c|c|c|c|c|}
\hline 21.05 .2013 & Brachycaudus (Prunaphis) cardui (L.) & 126 & 3 & 2.38 & 2 \\
\hline 21.05 .2013 & Dysaphis devecta (Walker) & 146 & 13 & 8.90 & 6 \\
\hline 21.05 .2013 & Hyalopterus pruni (Geoffroy) & 180 & 7 & 3.89 & 1 \\
\hline 22.05 .2013 & Dysaphis (Pomaphis) pyri (Boyer de Fonscolombe) & 125 & 3 & 2.40 & 1 \\
\hline 22.05 .2013 & Dysaphis (Pomaphis) pyri (Boyer de Fonscolombe) & 140 & 45 & 32.14 & 4 \\
\hline 22.05 .2013 & Eriosoma lanigerum (Hausmann) & 108 & 77 & 71.30 & 34 \\
\hline 22.05 .2013 & Hyalopterus pruni (Geoffroy) & 416 & 10 & 2.40 & 0 \\
\hline 28.05 .2013 & Dysaphis devecta (Walker) & 48 & 3 & 6.25 & 2 \\
\hline 28.05.2013 & Myzus cerasi (Fabricius) & 34 & 1 & 2.94 & 0 \\
\hline 03.06 .2013 & Hyalopterus pruni (Geoffroy) & 48 & 1 & 2.08 & 1 \\
\hline 04.06 .2013 & Hyalopterus pruni (Geoffroy) & 48 & 3 & 6.25 & 1 \\
\hline 04.06 .2013 & Hyalopterus pruni (Geoffroy) & 2200 & 47 & 2.14 & 23 \\
\hline 04.06 .2013 & Hyalopterus pruni (Geoffroy) & 56 & 2 & 3.57 & 1 \\
\hline 05.06 .2013 & Dysaphis (Pomaphis) pyri (Boyer de Fonscolombe) & 122 & 6 & 4.92 & 2 \\
\hline 05.06 .2013 & Hyalopterus pruni (Geoffroy) & 52 & 1 & 1.92 & 1 \\
\hline 10.06.2013 & Hyalopterus pruni (Geoffroy) & 290 & 8 & 2.76 & 1 \\
\hline 18.06.2013 & Hyalopterus pruni (Geoffroy) & 444 & 17 & 3.83 & 7 \\
\hline 18.06.2013 & Hyalopterus pruni (Geoffroy) & 433 & 12 & 2.77 & 6 \\
\hline 19.06.2013 & Dysaphis (Pomaphis) pyri (Boyer de Fonscolombe) & 488 & 100 & 20.49 & 75 \\
\hline 19.06.2013 & Dysaphis (Pomaphis) pyri (Boyer de Fonscolombe) & 680 & 72 & 10.59 & 44 \\
\hline 19.06.2013 & Dysaphis (Pomaphis) plantaginea (Passerini) & 315 & 35 & 11.11 & 19 \\
\hline 19.06.2013 & Eriosoma lanigerum (Hausmann) & 480 & 434 & 90.42 & 105 \\
\hline 19.06.2013 & Hyalopterus pruni (Geoffroy) & 360 & 6 & 1.67 & 1 \\
\hline 25.06 .2013 & Hyalopterus pruni (Geoffroy) & 41 & 3 & 7.32 & 0 \\
\hline 25.06 .2013 & Hyalopterus pruni (Geoffroy) & 1040 & 25 & 2.40 & 17 \\
\hline 25.06 .2013 & Hyalopterus pruni (Geoffroy) & 104 & 2 & 1.92 & 1 \\
\hline 25.06 .2013 & Hyalopterus pruni (Geoffroy) & 148 & 1 & 0.68 & 1 \\
\hline 16.07.2013 & Hyalopterus pruni (Geoffroy) & 79 & 9 & 11.39 & 2 \\
\hline 17.07.2013 & Aphis pomi De Geer & 115 & 11 & 9.57 & 2 \\
\hline 17.07.2013 & Eriosoma lanigerum (Hausmann) & 185 & 160 & 86.47 & 64 \\
\hline 17.07.2013 & Eriosoma lanigerum (Hausmann) & 68 & 23 & 33.82 & 11 \\
\hline 19.07.2013 & Hyalopterus pruni (Geoffroy) & 425 & 20 & 4.71 & 3 \\
\hline 22.07 .2013 & Brachycaudus (Prunaphis) cardui (L.) & 560 & 485 & 86.61 & 375 \\
\hline 05.09 .2013 & Eriosoma lanigerum (Hausmann) & 340 & 285 & 83.82 & 88 \\
\hline 02.10 .2013 & Eriosoma lanigerum (Hausmann) & 190 & 146 & 76.84 & 23 \\
\hline 23.10 .2013 & Eriosoma lanigerum (Hausmann) & 86 & 58 & 67.44 & 12 \\
\hline
\end{tabular}

Çalışma sonucunda çizelgeden de görüldüğü üzere genel parazitoitlenme oranı \%14.48 bulunmuştur. Örnek alınan tüm meyve türleri içerisinde yüzde parazitoitlenmenin en yüksek olduğu türler ise sirasiyla Dysaphis (Pomaphis) plantaginea (\%85.00), Eriosoma lanigerum (\%76.99), Microlophium carnosum (\% 64.29), Dysaphis (Pomaphis) pyri (\%12.01), Dysaphis devecta (\%10.75), Brachycaudus helichrysi (\%8.13), Hyalopterus pruni (\%4.87) olmuştur. Ayrıca en fazla parazitoitlenme 09.09.2012 tarihinde elma üzerinden elde edilen Eriosoma lanigerum'da olmuştur. Bu örnekte parazitoitlenme oranı \%92.68 olmuştur. Çalışmamızın 2013 yılı parazitoitlenme oranları Çizelge 4 'te verilmiştir.
Çizelge 4 incelendiğinde genel parazitoitlenme oranı \%18.79 bulunmuştur. Örnek alınan tüm meyve türleri içerisinde yüzde parazitoitlenmenin en yüksek olduğu türler ise sirasıyla Eriosoma lanigerum (\%72.87), Brachycaudus (Prunaphis) cardui (\%45.13), Dysaphis (Pomaphis) pyri (\%12.93), Dysaphis (Pomaphis) plantaginea (\%11.11), Aphis pomi (\%9.57), Dysaphis devecta (\%7.06), Hyalopterus pruni (\%3.90) olmuştur. Ayrica en fazla parazitoitlenme 19.06.2013 tarihinde elma üzerinden elde edilen Eriosoma lanigerum'da olmuştur. Bu örnekte parazitoitlenme oranı \%90.42 olmuştur. 


\section{Sonuçlar ve Tartışma}

Çalışma sonucunda ılıman iklim meyve türleri üzerinde Aphis pomi de Geer, Brachycaudus (Prunaphis) cardui (L.), Brachycaudus helichrysi (Kaltenbach), Chromaphis juglandicola (Kaltenbach), Dysaphis devecta (Walker), Dysaphis (Pomaphis) plantaginea (Passerini), Dysaphis (Pomaphis) pyri (Boyer de Fonscolombe), Eriosoma lanigerum (Hausmann), Hyalopterus pruni (Geoffroy), Myzus cerasi (Fabricius), Myzus (Nectarosiphon) persicae (Sulzer) olmak üzere toplam 11 yaprakbiti türü; sekonder konukçular üzerinde Brachycaudus (Prunaphis) cardui (L.), Brachycaudus helichrysi (Kaltenbach), Hyalopterus pruni (Geoffroy), Myzus (Nectarosiphon) persicae (Sulzer) olmak üzere toplam 4 yaprakbiti türü; bahçe içerisinde ve kenarında bulunan yabancl otlarda ise Aphis craccivora Koch, Microlophium carnosum (Buckton) ve Uroleucon sonchi (Linnaeus) olmak üzere toplam 3 yaprakbiti türü ve bu türlerin parazitoitlenme oranları tespit edilmiştir. Live ark. (2008), Xinjiang (Çin) pamuk alanlarında Acyrthosiphon gossypii Mordvilko, Aphis craccivora, A. gossypii ve M. persicae olmak üzere 4 yaprakbiti türünün bulunduğunu ve parazitlenmenin yüksek olduğunu ifade etmişlerdir. Alaserhat ve Canbay (2017), Erzincan ili biber alanlarında yapmış oldukları çalışma sonucunda konukçuları arasında ılıman iklim meyve türlerinin de olduğu Aphis craccivora Koch, Aphis fabae Scopoli, Aphis gossypii Glover ve M. (Nectarosiphon) persicae yaprakbiti türlerini ve bu türlerin parazitoitlenme oranları tespit etmişlerdir.

Çalışmanın yürütüldügü $\quad$ 2011-2013 yılları incelendiğinde örnek alının gerek meyve, gerek yabancı ot türleri içerisinde yüzde parazitoitlenmenin en yüksek olduğu türler ise sirasiyla E. lanigerum (\%74.93), M. carnosum (\% 64.29), D. (Pomaphis) plantaginea (\%42.85), $B$. (Prunaphis) cardui (\%29.39), D. (Pomaphis) pyri (\%10.46), A. pomi (\%9.57), D. devecta (\%9.37), B. helichrysi (\%8.13) ve $H$. pruni (\%4.46) olarak saptanmıștır. Dassonvilleve ark. (2013), çilek alanlarında yaygin olarak bulunan yaprakbiti türlerinin mücadelesinde parazitoitlerin kullanımı üzerine yürüttükleri çalışmada, çilek alanlarında 14 yaprakbiti türü ve 6 adet parazitoit tür tespit etmişlerdir. Parazitoitlerin çilek alanlarında bulunan yaprakbiti türleri içerisinde doğada yaygın olarak bulunan Acyrthosiphon malvae (Mosley), A. gossypii, Aulacorthum solani (Kaltenbach), Chaetosiphon (Pentatrichopus) fragaefolii (Cockerell, Macrosiphum euphorbiae (Thomas), Macrosiphum rosae (L.), M. persicae ve Rhodobium porosum (Sanderson) olmak üzere toplam 8 yaprakbiti türünde daha etkin oldukları, bu yaprakbiti türlerinde parazitlenmenin diğer türlere göre daha yüksek olduğu ifade edilmiştir. Live ark. (2018), Xinjiang (Çin) pamuk alanlarında yaprakbiti parazitizmi ve parazitoit çeşitliliği üzerine yapmış oldukları çalışma sonucunda birinci derecede önemli olarak belirtilen parazitoitlerden olan Binodoxys communis (Gahan) \%95.19, Praon barbatum Mackauer \%3.15, Trioxys asiaticus Telenga \%1.01 ve Lysiphlebus fabarum Marshall'un \%0.65 oranında doğada bulunduğunu ifade etmişlerdir.

Yllar itibarı ile genel parazitoitlenme oranlarına baktığımızda 2011 (\%6.3) yılı 2012 (\%14.48) ve 2013 (\%18.79) yllına göre en düşük, 2013 yllı ise diğer yılllara göre parazitoitlenmenin en fazla olduğu yll olarak tespit edilmiştir. Alaserhat ve Canbay (2017), Erzincan ili biber alanlarında 2012 ve 2013 yıllarında yapmış oldukları çalışma sonucunda sırası ile genel parazitoitlenme oranını $\% 21.98$ ve $\% 22.75$ olarak saptamışlardır. Live ark. (2018), Xinjiang (Çin) pamuk alanlarında 2016 ve 2017 yıllarında yapmış oldukları çalışma sonucunda yaprakbitlerinde parazitoitlenme oranlarının $\% 0$ 'dan \%26'ya kadar değişiklik gösterdiğini ve bu oranın tarla koşullarında yaprakbitlerinde gerçekleştirilen biyolojik mücadele çalışmalarında parazitoit kullanmanın düşük etkiye sahip olduğu anlamına geldiğini ifade etmişlerdir.

$\mathrm{Bu}$ çalışmayla Erzincan ve Gümüşhane illerinde yetiştirilen llıman iklim meyve türleri ve sekonder konukçularda bulunan yaprakbiti türleri ile bunların parazitoitlenme oranları belirlenmiştir. Ayrıca doğada var olan genel parazitoitlenmenin hangi yaprakbiti türlerinde daha fazla olduğu saptanmıştır. Biyolojik mücadele çalışmalarına temel oluşturacak veriler bu çalışma ile elde edilmiş ve ileride yaprakbitleri ile ilgili biyolojik mücadele çalışmalarında parazitoitlerin etkinliklerinin belirlenmesi, salım çalışmalarının yapılması gerektiği kanısına varılmıştır. Ancak yaprakbitleri ile parazitoitler arasındaki etkileşimin belirlenmesi amacıyla daha fazla sayıda ve detaylı çalışmaların yapılmasıyla kesin bir kanıya varılabileceği düşünülmektedir.

\section{Teşekkürler}

$\mathrm{Bu}$ çalışma Tarımsal Araştırmalar ve Politikalar Genel Müdürlüğü tarafından (TAGEM BS-11/0402/01-18) desteklenmiştir. Desteklerinden dolayı 
TAGEM'e teșekkür ederiz. Ayrıca Aphelinidae familyasına bağlı türün teşhisini yapan emekli öğretim üyesi Sayın Prof.Dr. Mikdat DOĞANLAR (Mustafa Kemal Üniversitesi, Bitki Koruma Bölümü)'a, Braconidae familyasına bağlı türlerin teşhisini yapan Sayın Doç.Dr. Coşkun GÜÇLÜ (Eskişehir Osmangazi Üniversitesi, Ziraat Fakültesi, Tarımsal Biyoteknoloji Bölümü)'ye ve Aphididae familyasına bağlı türlerin teşhislerinde yardımcı olan Sayın Dr. Işıl ÖZDEMİR (Ankara Zirai Mücadele Merkez Araştırma Enstitüsü)'e teşekkür ederiz.

\section{Kaynaklar}

Alaserhat, İ., \& Canbay, A. (2017). Aphididae species, their parasitoids, predators, and parasitism rates on pepper (Capsicum annuum L.). Entomological News, 127(1), 36-50.

Alaserhat, İ. (2019). Erzincan ili elma ağaçlarında bulunan zararlı ve faydalı böcek türleri ile bazı önemli zararlı türlerin doğada görülme zamanı. Avrupa Bilim ve Teknoloji Dergisi, 17, 1116-1124.

Anonim, (2008). Zirai Mücadele Teknik Talimatları. T.C. Gıda, Tarım ve Hayvancılık Bakanlığı Tarımsal Araştırmalar Genel Müdürlüğü, Cilt:4, $388 \mathrm{~s}$, Ankara.

Anonim, (2013). Bitkisel Üretim İstatistikleri. Türkiye İstatistik Kurumu, https://biruni.tuik.gov.tr/ medas $/$ ?kn=92\&locale $=\operatorname{tr} \quad$ (Erişim tarihi 03.03.2020).

Anonim, (2019). Bitkisel Üretim İstatistikleri. Türkiye İstatistik Kurumu, https://biruni.tuik.gov.tr/ medas $/$ ?kn=92\&locale $=$ tr $\quad$ (Erişim tarihi 03.03.2020).

Aslan, B., \& Karaca, İ. (2005). Fruit tree aphids and their natural enemies in Isparta region, Turkey. Journal of Pest Science, 78, 227-229.

Bissel, T.L. (1978). Aphids on Juglandaceae in North America. University of Maryland Agricultural Experiment Station Contribution, 911, 78 p, Maryland, USA.

Blackman, R.L., \& Eastop, V.F. (1984). Aphids on The World's Crops: An Identification quide. A Wiley. Intenscience Publication, 466 p, London, England.

Blackman, R.L., \& Eastop, V.F. (1994). Aphids on The World's Trees: An Identification and information quide $\mathrm{CAB}$ International. Department of Entomology the Natural History Museum, 986+16 p, London, England.

Blackman, R.L., \& Eastop, V.F. (2000). Aphids on The World's Crops: An Identification quide. Second Edition. A Wiley. The Natural History Museum Intenscience Publication, 414 p, London, England.
Blackman, R.L., \& Eastop, V.F. (2020). Aphids of The World's Plants. An online identification and information guide. http://www.aphidsonworldsplants.info/Introducti on.htm (Access date 05.03.2020).

Bodenheimer, F.S., \& Swirski, E. (1957). The Aphidodea of the Middle East. The Weizmann Science Press of Israel, 378 p, Jerusalem, Israel.

Bora, T., \& Karaca, İ. (1970). Kültür Bitkilerinde Hastalığın ve Zararın Ölçülmesi. Ege Üniversitesi Ziraat Fakültesi yardımcı ders kitabı. No: 167, 3-43, İzmir.

Dassonville, N., Thiellemans, T., \& Gosset, V. (2013). FresaProtect and BerryProtect: mixes of parasitoids to control all common aphid species on protected soft fruit crops Product development and case studies from three years of experience. Aspects of Applied Biology, 119, 79-88.

Daşcı, E., \& Güçlü, Ș. (2008). Iğdır ovasında meyve ağaçlarında bulunan yaprakbiti türleri (Homoptera: Aphididae) ve doğal düşmanları. Atatürk Üniversitesi Ziraat Fakültesi Dergisi, 39(1), 71-73.

Düzgüneș, Z., \& Toros, S. (1978). Ankara ili ve çevresinde elma ağaçlarında bulunan yaprakbiti türleri ve kısa biyolojileri üzerinde araștırmalar. Türkiye Bitki Koruma Dergisi, 1(3), 151-175.

Eastop, V.F. (1971). Keys for the identification of Acyrtosiphon (Hemiptera: Aphididae). Bulletin of British Museum (Natural History) Entomology, 26(1), 1-115.

Eastop, V.F. (1972). A taxonomic review of the species of Cinara curtis occurring in Britain (Hemiptera: Aphididae). Bulletin of British Museum Entomology (Natural History), 27(2), 1-186.

Eastop, V.F., \& Hille Ris Lambers, D. (1976). Survey of the World's Aphids. The Hague: W. Junk. pp: 573.

Hille Ris Lambers, D. (1945). De Bloedvlekkenluis van Appel, Sappaphis devecta (Wlk). Tijdschr. Ov. Plantenziekt, 51, 57-66.

Hille Ris Lambers, D. (1947a). Contributions to a Monograph of the Aphididae of Europe. III. Temminckia, 7, 179-319.

Hille Ris Lambers, D. (1947b). On some mainly Western European aphids. Zoologische Mededeelingen, 28, 291-333.

Hille Ris Lambers, D. (1949). Contribution to a monograph of the Aphididae of Europe. Temminckia, 3, 282285.

Hille Ris Lambers, D. (1950). On mounting aphids and other soft skinned insects. Entomologische Berichten, 13, 55-58.

Hille Ris Lambers, D. (1969). Four new species of Cavariella del Guercio, 1911 (Homoptera: 
Aphididae). Estratto Dalle Memorie Della Sociate Entomologica Italiana, 48, 285-299.

Hille Ris Lambers, D. (1973). Notes on some oriental aphids. Orient. Insects, 7, 239-258.

Görür, G. (2004). Aphid (Homoptera: Aphididae) species on pome fruit trees in Nigde Province of Turkey. Turkish Journal of Entomology, 28(1), 21-26.

Gürbüz, N.A. (2001). Niğde ve Yöresinde Yumuşak Cekirdekli Meyvelerde Zararlı Olan Afit (Insecta: Homoptera: Aphidoidea) Türlerinin Belirlenmesi. Niğde Üniversitesi Fen Bilimleri Enstitüsü Bitki Koruma Anabilim Dalı, Basılmamış Yüksek Lisans Tezi, 68 s., Niğde.

Li, H.B., Wu, K.M., Xu, Y., Yang, X.R., Yao, J., \& Sun, S.L. (2008). Dynamic analysis of population of cotton aphids in the south of Xinjiang. Xinjiang Agricultural Sciences, 45, 670-675.

Li, J., Wu, Y., Zhang, Q., Li, H., Pan, H., Lu, W., Wang, D., Zhang, J., \& Lu, Y. (2018). Aphid parasitism and parasitoid diversity in cotton fields in Xinjiang, China. PLoS One, 13(11), 1-14.

Kaplan, M. (2019). Diyarbakır ili bazı kiraz bahçelerinde bulunan zararlı ve faydalı böcek türleri ile bazı önemli zararlı türlerin doğada görülme zamanı. Avrupa Bilim ve Teknoloji Dergisi, 17, 283-289.

Lazarov, A., \& Grigorov, P. (1961). Karantina na Rastenijata. Zemizdat. Sofia. p. 258.

Lodos, N. (1986). Türkiye Entomolojisi II (Genel, Uygulamalı ve Faunistik). Ege Üniversitesi Ziraat Fakültesi Yayınları No:429, 580 s., İzmir.

Matheus, R.E.F. (1993). Diagnosis of Plant Virus Diseases. CRS Press Inc, 374 p, Boca Raton, Florida.

Narmanlıoğlu, H.K., \& Güçlü, Ş. (2008). İspir (Erzurum) ilçesinde meyve ağaçlarında bulunan yaprakbiti türleri (Homoptera: Aphididae) ve doğal düşmanları. Atatürk Üniversitesi Ziraat Fakültesi Dergisi, 39(2), 225-229.

Ölmez Bayhan, S., \& Ulusoy, M.R. (2002). Diyarbakır İlinde Aphidoidea Üst familyasına Bağlı Türlerin Predatörlerinin Belirlenmesi, 463 s. Türkiye V. Biyolojik Mücadele Kongresi Bildirileri (4-7 Eylül, 2002, Erzurum), 237-246 s.

Ölmez Bayhan, S., \& Özdemir, I. (2009). Güneydoğu Anadolu Bölgesi'nde Aphidoidea (Hemiptera) Türleri, Konukçuları ve Doğal Düşmanları Üzerinde Araştırmalar (Sonuç Raporu) (Yayınlanmamış). Tarımsal Araştırmalar Genel Müdürlüğü, Ankara.

Özbek, H., Güçlü, Ş., Hayat, R., \& Yıldırım, E. (1998). Meyve, Bağ ve Bazı Süs Bitkileri Zararlıları. Atatürk Üniversitesi Ziraat Fakültesi Yayınları No: 323, 357 s, Erzurum.
Praslicka, J., \& Huszar, J. (2005). Hymenopteran parasitoids (Hymenoptera, Aphidiidae) on cereal aphids (Sternorrhyncha, Aphidoidea) in integrated and ecological pest management systems. Biologiasection Zoology, 60(2), 227229.

Remaudiere, G. (1954). Deuxieme addition a la liste des Dactynotina eve Myzinae (Hem. Aphidoidea) de la faune Française. Revue de Pathologie végétale vé d'entomologie Agricole de France, 4, 232-240.

Remaudiere, G., \& Remaudiere, M. (1997). Catologue des Aphididae du Monde (Of the World's Aphididae) Homoptera, Aphidoidea, Preface Par V.F. Eastop, INRA Editions, pp: 473.

Shaposhnikov, G.K. (1964). Suborder Aphidinea-Plant Lice. (In Keys to the Insects of the European Part on the USSR. Editor: G. Bei-Bienko. Moscow and Leningrad, Russian, 616-799.

Stroyan, H.L.G. (1961). Identification of aphids living on Citrus. FAO Plant Protection Bulletin, 9(4), 45-65.

Stroyan, H.L.G. (1963). The British Species of Dysaphis Börner (Sappaphis auctti nec Mats.) Part II. Her Majestry's Stationery Office, 119 p, London, England.

Stroyan, H.L.G. (1977). Hemiptera, Aphidoidea (Part), Chatophoridae and Callaphidae. Handbooks for the Identification of British Insects. II, Part 4 (a). Royal Entomology Society of London, 130.

Stroyan, H.L.G. (19849. Aphids-Pterocommatinae and Aphidinae (Aphidini) Hemiptera: Aphididae. Handbooks for the Identification of British Insects. Royal Entomology Society of London, 2(6), 232.

Toros, S. (1973). Bitki patojen viruslerinin aphidlerle nakil mekanizması. Bitki Koruma Bülteni, 13(2), 83-105.

Tuatay, N., \& Remaudiere, G. (1964). Premiere contribution au catalogue des Aphididae (Homoptera) de la Turquie. Rev. Path. Vegveale et D'entomologie Agricole de France, 43(4), 243-278.

Ülkümen, L. (1973). Bağ-Bahçe Ziraatı. Atatürk Üniversitesi Ziraat Fakültesi Yayınları No:128, 404 s, Erzurum.

Yoldaş, Z., Koçlu, T., \& Güncan, A., (2007). İzmir İlinde Turunçgillerde Bulunan Yaprakbiti Türleri ile Doğal Düşmanları Arasındaki İlişkiler, 342 s. Türkiye II. Bitki Koruma Kongresi, (27-29 Ağustos 2007, Isparta), $21 \mathrm{~s}$.

Yumruktepe, R. (1993). Doğu Akdeniz Bölgesi Turunçgil Bahçelerinde Zararlı Olan Yaprakbiti (Hemiptera: Aphidiade) Türleri, Tanınmaları, Yayılışları, Doğal Düşmanları, Popülasyon Dalgalanmaları ve Kimyasal Mücadelesi Üzerinde Araștırmalar. Çukurova Üniversitesi Fen Bilimleri Enstitüsü Bitki Koruma Anabilim Dalı, Basılmamış Doktora Tezi, 127 s., Adana 